Canadian Oncology

Nursing Journal

Revue canadienne

de soins infirmiers

en oncologie

Volume 28, Issue 1 • Winter 2018

elSSN: 2368-8076 


\title{
Bridging the gap from the oncology setting to community care through a cross-Canada environmental scan
}

\author{
by Danielle M. Wittal
}

\begin{abstract}
Due to the growing number of cancer survivors, there is a need to evaluate the provision of follow-up care to ensure the needs of patients are met. An environmental scan was conducted provincially and nationally, which aimed to gain a broader understanding of survivorship care, highlight any trends, and report disparities in care during the transition from the oncology to the community setting. The environmental scan involved gathering data from nine out of 10 provinces through telephone interviews, seeking relevant documentation, and identifying areas of good practice. There was a wide variation in practice across the provinces and some recommendations for improving care are made. Findings emphasize the need to promote increased patient self-management and self-advocacy. Implications from the study are that cancer survivorship is a complex topic that requires communication and coordination across services to ensure a seamless transition.
\end{abstract}

\section{INTRODUCTION}

$\mathrm{T}$

The prevalence of cancer is increasing by three percent per 1 year in British Columbia alone (British Columbia Cancer Agency, 2015), with two out of five Canadians expected to be diagnosed in their lifetime (Canadian Cancer Society, 2015). Survivor statistics for British Columbia indicate the number of cancer survivors is anticipated to be 250,000 by 2020, with 196,900 people being diagnosed nationally in 2015 (Canadian Cancer Society, 2015). Approximately 22.4 million cancer survivors are expected globally (National Cancer Institute, as cited in Grunfeld, 2006). Without a dramatic culture shift, the current health care system will not be able to sustain access and provision to high quality cancer care following treatment. There is a growing emphasis on self-management and self-advocacy (including a strong self-concept/self-confidence, improving patient empowerment, increasing self-esteem, fostering a sense of control, and assisting adaptation to a life with cancer) as it has been shown to carry many positive benefits for survivors (Hagan \& Donovan, 2012; Wiljer et al., 2013). However, currently there is no local or national policy context for survivorship care in Canada.

\section{ABOUT THE AUTHOR}

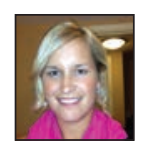

Danielle M. Wittal (Principal Researcher), Radiation

Therapist (RTT), Masters of Science (MSc), British Columbia

Cancer Agency, Centre for the Southern Interior Kelowna, British

Columbia, 2118 Crosshill Drive, Kamloops, BC V1S 0C6

Danielle.wittal@bccancer.bc.ca; dwwittal@gmail.com

Tel: 778-220-8822

\section{PURPOSE}

This project aimed to inform priority areas for focus in a wider study aligned with the British Columbia Cancer Agency Provincial Survivorship and Primary Care Program strategic priorities. Conducting an environmental scan helped to understand areas of strengths and weakness in the transition of care processes across the country, and suggested recommendations for a seamless transition to the community setting within British Columbia. This paper aims to provide a snapshot of the transition of care practices across Canada. It is my hope that it will help inform local developments and lead to evidence-based solutions for high quality cancer care.

\section{METHODS}

\section{Context}

The environmental scan of jurisdictional practices across Canada was conducted from May 2015 to September 2015, with nine out of 10 provinces participating. The project consisted of two parts, a literature review and a series of interviews.

\section{Data Collection}

The purpose of the environmental scan was to gain an understanding of what is happening provincially and nationally, and to identify activity, events, trends, issues and expectations regarding the transition of care from the oncology setting to community care. The scan consisted of 1 ) a literature review, and 2) a series of interviews across the country that included individuals in the following types of roles: lead navigator for survivorship research, oncology nurse advisor, director of supportive care, manager of survivorship program, lead for person-centred care integration, and patient care navigators. Thematic analysis was employed for this study. This approach was selected as the most suitable analysis for identifying overall themes following careful reading and re-reading of documents and interviews (Daly, Kellehear, \& Gliksman, as cited in Fereday \& Muir-Cochrane, 2006).

By utilizing a combination of codified and tacit knowledge, while employing a passive approach to data collection, local ethical or governance approval was not required. Ethical approval was granted from Sheffield Hallam University for the study, as this work was part of my graduate degree. Participating in the telephone interviews implied informed consent. There was no physical, psychological or emotional harm for the participants and anonymity of each was honoured. 


\section{Search Strategy for Literature Review}

Interfaces searched included Ovid and EBSCOhost to incorporate databases CINAHL complete, MEDLINE with full text, PubMed-NCBI, EBM (Cochrane) \& EMBASE and PsycInfo.

Terms searched consisted of transition of care/transition, oncology, continuity of care/organization \& administration, survivorship, communication, general practitioner, physician's role, patient-physician relations, physician's practice patterns, health services needs and demands, physicians/psychology, patient care, cancer survivors/survivors, neoplasms, follow-up, care plans, Canada, British Columbia, Alberta, Saskatchewan, Manitoba, Ontario, Quebec, Newfoundland, Prince Edward Island, Nova Scotia and New Brunswick.

Inclusion criteria: initial searches 2005-01-01 to 2015-12-31, however, included other pertinent articles as early as 1993; published data; grey literature.

Exclusion criteria: pediatric/children, Medline records.

Initially the search included only Canadian articles, however more information was needed so the search was opened up to provide more support, and gather an understanding of what is happening globally.

The completed literature review pre-defined themes that were subsequently supported by the findings in the interviews. The themes identified in the literature review were roles and responsibilities; type and timing of information; and models of care. Delineating who is responsible for what care of the patient and providing pertinent information at the appropriate time are crucial to enabling the primary care physician to assume exclusive responsibility for follow-up care. Implementing the appropriate model of care, best suited to each health region in Canada, and utilizing a survivorship care plan in practice, offer feasible solutions to the identified needs mentioned in this review.

\section{Interview Participants}

Participants targeted for interview included lead navigators for survivorship research, oncology nurse advisors, directors of supportive care, managers of survivorship programs, leaders for person-centred care integration, and patient care navigators. Resources within the British Columbia Cancer Agency Provincial Survivorship and Primary Care Program, the Canadian Partnership Against Cancer, and internet searching identified a starting point of contact in each province. An email was sent to each contact to explain the purpose of the project and arrange a time for an interview. If the initial person contacted was not the most knowledgeable about the study subject, then I was referred to another source. I often had to contact three or four different people within a province to find the ideal participant.

The interviews were carried out by the principal researcher and followed a series of questions (see Table 1). Field notes were made, as well. After the initial interview, further discussion took place via telephone and/or email if clarification was needed or additional documents were required. The appropriate documents were obtained from each jurisdiction and included transfer of care/discharge letters, discharge packages, survivorship care plans, and patient/primary care physician checklists for follow-up recommendations. Documents were included only for reference and to provide a guide/idea for change across Canada.

\section{Table 1: Interview Guide Questions}

\section{Questions asked for the environmental scan}

1. What is your current practice regarding transition to community care of oncology patients who are actively finished treatment? Is primary care now the responsibility of the oncologist or primary care provider? What kind of information is provided to the patient and primary care giver at the time of transition?

2. Is there provincial standardization regarding the process and outcomes of transition of care?

3. Did you hold focus groups/engagement sessions/pilot project to provide the necessary information for transition of care? Did you include primary care physician and specialist oncologists?

4. If you have a survivorship care plan in practice, what does the care plan look like? What information does it provide the patient and primary care provider?

5. Who is responsible for process of transitioning patients to primary care physicians? Is this information given offhand or is there an official meeting? If so - who is involved?

6. If a survivorship care plan is in place, is this a paper document or electronic or both?

\section{RESULTS}

\section{Variations in Practice at Discharge}

The standard of practice across the country for transition of care from the oncology setting to the community is highly variable. In general, there are no provincial standards or guidelines within each province regarding follow-up of cancer survivors, as it is challenging to gain consensus among specialist oncologists. There was wide variation in practices for follow-up care within each regional cancer centre, specialist oncologist and/or disease site within each province, with the exception of Manitoba. At the very least, each province has some form of communication to the primary care physician including a dictation letter and/or transfer of care letter, sent out either electronically or via mail. However, the information within those letters is generally not standardized. For all provinces, the specialist oncologist is responsible for discharging patients back to primary care.

\section{Examples of Good Practice}

Good practice was defined by the primary author as an idea in place or physical handout designed to improve the transition process from the oncology setting to the community. The themes in the literature concerning gaps in transition care, which included roles and responsibilities, type and timing of information, and models of care, were clearly identified and supported by the interviewees. Examples of good practice identified from the interviews can help to bridge the gaps associated with the recognized themes and are described below. 


\begin{tabular}{|c|c|c|c|}
\hline $\begin{array}{l}\text { British } \\
\text { Columbia }\end{array}$ & $\begin{array}{l}\text { No provincial standard and varies by regional cancer } \\
\text { centre, oncologist and disease site. The oncologist } \\
\text { is responsible for discharge letter that is sent to the } \\
\text { primary care physician. }\end{array}$ & $\begin{array}{l}\text { Future work includes exploring the } \\
\text { transition of care from oncology to } \\
\text { the community. }\end{array}$ & Not currently in practice. \\
\hline Manitoba & $\begin{array}{l}\text { Highly variable at this time. Depends if the patient } \\
\text { has a primary care physician and the relationship with } \\
\text { their primary care physician. Have a transition clinic } \\
\text { appointment occurring anywhere from } 6 \text { weeks to } 3 \\
\text { months after active treatment is complete, which isn't } \\
\text { always utilized. Patients are given individualized care } \\
\text { plan with a copy faxed to primary care physician. }\end{array}$ & $\begin{array}{l}\text { Pilot project completed which led } \\
\text { to informal discussions to inform } \\
\text { treatment summaries and disease- } \\
\text { specific survivorship care plans. }\end{array}$ & $\begin{array}{l}\text { Currently in place for colorectal, } \\
\text { lymphoma, ovarian, peritoneal and } \\
\text { fallopian tube cancers. Leukemia, } \\
\text { bone marrow transplant and } \\
\text { thoracic are expected to launch } \\
\text { in } 2016 .\end{array}$ \\
\hline Quebec & NA & NA & NA \\
\hline New Brunswick & $\begin{array}{l}\text { No provincial standard, varies clinic to clinic. The } \\
\text { treating oncologist is responsible for transition of care. } \\
\text { Dictation is sent to the primary care physician, not in } \\
\text { any standardized form. }\end{array}$ & Nothing currently. & Not currently in practice. \\
\hline Nova Scotia & $\begin{array}{l}\text { No provincial standard, varies clinic to clinic. Transfer } \\
\text { of care letters are given to the patient and primary } \\
\text { care physician along with personalized dictated notes. }\end{array}$ & $\begin{array}{l}\text { In } 2010 \text {, a consultant firm was } \\
\text { hired to hold focus groups and } \\
\text { interviews with specialist oncologists } \\
\text { and primary care physicians. These } \\
\text { helped to dictate and inform the } \\
\text { transfer of care letters. }\end{array}$ & $\begin{array}{l}\text { Currently set up for colorectal, } \\
\text { breast and thyroid disease sites } \\
\text { but they are not individualized. }\end{array}$ \\
\hline $\begin{array}{l}\text { Prince Edward } \\
\text { Island }\end{array}$ & $\begin{array}{l}\text { No provincial standard and is dependent upon the } \\
\text { oncologist. Consult letter is provided to the primary } \\
\text { care physician if follow-up care is to be provided by the } \\
\text { primary care physician. }\end{array}$ & Nothing thus far. & Not currently in practice. \\
\hline Newfoundland & $\begin{array}{l}\text { No provincial standard and depends if the patient } \\
\text { is receiving adjuvant therapy. Patient and primary } \\
\text { care physician are each given a written care plan that } \\
\text { includes recommended testing and follow-up care } \\
\text { guidelines. }\end{array}$ & $\begin{array}{l}\text { Nothing thus far, however the social } \\
\text { work department offers a transition } \\
\text { workshop help twice yearly. }\end{array}$ & $\begin{array}{l}\text { Currently in place for breast and } \\
\text { colorectal disease sites. }\end{array}$ \\
\hline
\end{tabular}




\section{Checklist}

The patient and primary care physician checklists that Alberta employs provides an option to support primary care physicians to feel better equipped and confidently handle surveillance regarding cancer recurrence for all disease site groups. The checklist includes a clinical exam every six months for the first two years, then yearly from three to five years; and the diagnostic exam, yearly one to five years. The checklist provides a place to note the date of the exam and findings to help the patient keep track of when appointments should be and to aid the primary care physician with best follow-up care practices. Nova Scotia utilizes a primary care physician feedback note that accompanies the transfer of care letter. This is a great tool that allows primary care physicians to have a voice and share their thoughts regarding information received and needed at the time of transition. It is seen as a help to primary care physicians in effectively caring for oncology patients. The patient and primary care physician checklists for follow-up care and primary care physician feedback notes are tools that can be adopted to help narrow the gap between oncology and the community.

\section{Transition Appointment}

In Manitoba, the current transition of patients occurs through a transition appointment that is built into clinical practice and done consistently. A transition appointment is created and booked in the clinic once a patient has finished treatment and is starting the follow-up/surveillance phase. The primary provider of care may not change, but the transition appointment is always completed. The transition appointment is provided by the patient's oncologist and includes screening for distress, appropriate referrals, and the provision of a personalized written treatment summary and survivorship care plan to the patient (with copies to the primary care physician and surgeon). The transition appointment occurs after the first follow-up screening (CT scan for colorectal, mammogram for breast) and can happen anywhere from six weeks to approximately three months after the patient leaves active treatment. At this appointment, patients are given their individualized care plan for their own records with a copy faxed to the primary care physician.

Current practice in Ontario varies by regional cancer centre, but follows one of three models for transitions of breast and colorectal cancer survivors. The models include patients going (1) directly to the primary care physician; (2) to a transition clinic led by a nurse practitioner, advanced practice nurse and/or a general medical oncologist for one to three visits before transition to their primary care physician; or (3) shared care that includes visits with both the primary care physician and medical, radiation, and/or surgical oncologists for a specified period of time. The information provided to the patient and primary care physician also varies by region with all regions including some variation of a survivorship care plan or treatment summary. For regions with a transition clinic, patients are scheduled for a transition clinic appointment, which includes an explanation of the care plan and time for pending questions. Ultimately, the oncologist is responsible for identifying appropriate cancer survivors for transition. The specific process and providers involved vary by region and depends on the accepted model the region has implemented.

\section{Transition Coordinator}

In Saskatchewan, the Saskatchewan Cancer Agency is currently working on a project that includes the development of a transition coordinator position. The transition coordinator program is based on a patient navigator program in which the ideal candidate is a social worker. The goal of the transition coordinator role is to support patients to manage their own care by providing them with information on community programs and available resources within the region/area where they live. The transition coordinator will screen and assess the patient and the family's ability and requirements to coordinate services and address their supportive care needs. Patients discharged from care will be contacted by telephone within two weeks of their discharge date. Assessments will be conducted over the phone and will address any concerns they may have in the following areas: fear of recurrence, chronic pain, mental or physical fatigue, lymphedema, changes in relationships and sexual function, issues with altered body image, dietary needs / changes, health or life insurance matters, financial difficulties, employment issues, depression or anxiety, uncertainty about the future, questioning sense of purpose in life, social isolation, and lack of communication/coordination between healthcare providers.

Table 3: Goals and objectives of the Transition Coordinator role

- Navigate a complex and somewhat fragmented care system as it links with community-based resources.

- Empower patients to mobilize community resources. This will be accomplished with a strategy of empowerment where with information, connection, and decision support, patients will be directing their own care.

- Single person of contact/support at the end of the treatment phase and into the survivorship phase to aid in continuity of care.

- The community-based focus of the transition coordinator means that they will work with patients' families, community groups and organizations, to identify and build strong community links and capacity to meet the follow-up needs of cancer survivors.

- The role of the general practitioner/primary care provider will be strongly supported with assistance provided to patients to enhance and maintain their relationship with their general practitioner especially in the rural environment. Assistance will be provided to patients to obtain a general practitioner should they not have one.

- The transition coordinator will be responsive, flexible and aware, as the needs of individual patients and the communities in which they live can change in the cancer care pathway. Continuous communication with stakeholders will aid in identifying changing needs. 


\section{Survivorship Workshops}

Saskatchewan provides a transition survivorship program offered for patients after treatment that covers some basic survivorship information. In Ontario, some regional cancer centres provide after-cancer classes for survivors during the transition period and/or patient-specific material on various survivorship issues (i.e., potential side effects or late effects, information on proper nutrition and healthy lifestyle). Prince Edward Island offers a number of different programs including Look Good Feel Better (nationally run program that gives you tools on how to look and feel better during your treatments) and a cancer transition program (three-hour program designed for cancer patients who have finished active treatment and includes discussion of follow-up care, healthy eating, physical activity, coping and adjustment, survivorship and community resources). Nova Scotia holds monthly sessions called Living Beyond Cancer, a PowerPoint presentation, which is run by nurses, radiation therapists, chemotherapy nurses, nutrition, psychosocial, social work and spiritual care. YouTube videos are included and the program is available via telehealth if patients are unable to attend in person. Also offered is a provincial program designed to help people manage chronic illness entitled, "Your Way to Wellness". Additionally, offered twice yearly, is a Cancer Transitions group in which patients are invited to participate. If there is anticipated trouble regarding transitioning (i.e., anxiety) patients may be referred by the physician or the Navigator group to the program and can still be followed by the navigator, as well as the social work department. This workshop is designed to assist people moving from active care back to their "normal" life and focuses on a number of topics over a six- to 10 -week program. Patients are also able to access the patient navigation service at any time during their cancer journey, from the time of suspicion of a cancer diagnosis through to survivorship.

\section{DISCUSSION}

With the growing number of cancer survivors, follow-up care with specialist oncologists will not be able to be maintained for everyone. Hence there is a need to shift follow-up care to primary care physicians, as is appropriate for selected disease sites.

The literature review revealed some key findings regarding barriers to the exclusive transfer of care from the oncology setting to the community. These barriers include lack of role clarity/role delineation, debates regarding the best follow-up strategy, differing perspectives on who should provide what care; and the type and timing of information (Grunfeld \& Earle, 2010; Cheung et al., 2013; Del Guidice, Grunfeld, Harvey, Piliotis \& Verma, 2009; Haq et al., 2013; Mao et al., 2009; McCabe \& Jacobs, 2012; Nissen et al., 2007). The interface between primary care physician and specialist oncologists is where gaps exist at present. This project was designed with the aim of making recommendations to help bridge this gap.

\section{Role Delineation}

It is recognized by Cheung et al. (2013) and supported by other authors (Ackerman et al., 2014; Dawes et al., 2015; Del
Guidice et al., 2009; Nissen et al., 2007; Salz et al., 2012) that primary care physicians may not be equipped with the knowledge, education, and experience to confidently handle issues and provide adequate follow-up care (to include surveillance testing for cancer recurrence) for complex disease groups. However, many studies in Canada and from other parts of the world have shown that the primary care physician remains the primary coordinator of care during cancer survivorship, and that care can effectively be provided by the primary care physician for low-risk cancer groups (Del Guidice et al., 2009; Mao et al., 2009; Grunfeld et al., 1995, 1996, 1999, 2006; Sisler, Brown \& Stewart, 2004; Wattchow et al., 2006; Wood, 1993).

Ye, Cheung, Goddard, Horvat and Olson (2015) found that most radiation oncologists follow the more complex disease site groups (Central Nervous System, gastrointestinal, head and neck, gynecological and genitourinary patients) due to treatment-related toxicity and the potential for further treatment; the oncologists believed that follow-up by the primary care physician required additional training and more guidelines. Research completed in Nova Scotia supported this viewpoint, which noted primary care physicians are unclear about who is responsible for what follow-up care. In Alberta, nurse practitioners often provide follow-up care post-cancer treatment, and regularly determine and implement transitions to primary care physicians. Interestingly, Saskatchewan puts great emphasis on the involvement of the primary care provider throughout the cancer care journey and does not discharge the patient from cancer care unless it is confirmed that they have a primary care physician. The involvement of the primary care physician throughout the cancer care journey can lead to increased trust in the primary care physicians' ability to assume responsibility for follow-up care, as they are constantly informed about their patient (Urquhart, Folkes, Babineau \& Grunfeld, 2012). Manitoba does not discharge patients unless it is confirmed that they have a primary care provider and a decently established relationship with their primary care physician. Statistics Canada (2014) noted that in 2013 15.5\% of Canadians 12 years and older do not have a primary care physician. This is an important aspect and should be considered in regards to how any province handles transitioning patients when they do not have a confirmed primary care physician.

\section{Perspectives on Care}

Historically, cancer surveillance has been provided by specialist oncologists while the primary care physicians focus on preventative interventions directed at non-cancer conditions (Earle, Burstein, Winer \& Weeks, 2003; Earle \& Neville, 2004; Snyder et al., 2008). Sisler et al. (2004) examined how colorectal survivors in Manitoba evaluated their quality of follow-up care after treatment versus those in the care of a primary care physician. Results showed that patients followed by a primary care physician evaluated their transition of care after treatment favourably, especially when there were no complications following active treatment. It was noted that those who encountered more challenges in their recovery would have an increased need for more information and support services to have a smoother transition. Manitoba utilizes a survivorship 
care plan that contains a discharge letter, follow-up recommendations, and a personalized cancer treatment summary. The discharge letter states in bold whom is responsible for follow-up care (the specialist oncologist or primary care physician), prescriptions, and future routine investigations.

To ensure a seamless transition from the oncology setting to the community, primary care physicians need to be supported and educated if they are to have the capability of providing ongoing cancer surveillance. Urquhart et al. (2012) noted that the transition from active treatment to routine follow-up can be overwhelming to survivors, as the shift in personal responsibility can lead to feelings of distress and abandonment. Del Guidice et al. (2009) support this observation with findings from their study; primary care physicians have concerns that patients expect follow-up care to be completed by their oncology specialist. Patients valued the participation of their primary care physician in follow-up care, but many liked to receive reassurance from the specialist oncologist, preferring follow-up care through the specialists rather than their primary care physician. Patients also shared the concerns that primary care physicians may lack knowledge, competencies related to cancer care, and the ability to access specialist care and supports if needed. This underpins the need to better educate the primary care sector on survivorship care issues and include them in the cancer care pathway. Ultimately, patients, specialist oncologists and primary care physicians all need to have confidence in primary care physicians to resume full responsibility for post-cancer care. The emphasis that Saskatchewan places on the involvement of the primary care physician throughout the cancer care journey is important in helping the patient and specialist oncologist to have the confidence and increased trust in the primary care physicians' ability to assume responsibility for post-cancer care.

\section{Type and Timing of Information}

The type and timing of information given to patients and to primary care physicians was noted as an important aspect for the primary care physician and a gap in current transition of care practices across British Columbia and in the literature (Del Guidice et al., 2009; Haq et al., 2013). The type of information given to patients varies greatly across the country and generally there is no standardization within any single province. In most provinces, each cancer clinic has its own wealth of information, which can be found in a library setting, physical patient handouts/pamphlets or online. Information includes patient-specific material on various survivorship issues (i.e., potential side effects or late effects, information on proper nutrition, healthy lifestyle, medication, psychosocial and emotional effects).

It is recognized that the timing of giving patient information (as soon as the patient exits the oncology setting) to the primary care physician is crucial to enable primary care physicians to have meaningful post-cancer care discussions with patients (Haq et al., 2013). Generally, across all provinces the goal is to provide information to the primary care physician (whether it be a dictation letter and/or transfer of care letter or survivorship care plan) at the time of discharge. Utilizing the patient and family physician checklists, transition coordinator, transition appointment and/or survivorship care plan (which ought to include patient-specific letters from specialists, clear patient-specific guidelines on follow-up practices, expedited access to investigations and re-referral to specialty care) are all tools to provide information and a smooth transition for patients at the time of discharge (Del Guidice et al., 2009; Smith, Singh-Carlson, Downie, Payeur \& Wai, 2011; Ye, Cheung, Goddard, Horvat \& Olson, 2015).

\section{RECOMMENDATIONS}

Improving the current transition of care practices across British Columbia and Canada as a whole, while ensuring high-quality cancer care, requires change. This exploration identified gaps in care, which included role delineation, provider of care discrepancies, and information needs and requirements. Discussion yields four recommendations including implementation of a 1) follow-up checklist, 2) transition appointment, 3) transition coordinator, and/or 4) survivorship care plan. The transition appointment and/or transition coordinator can be considered possible first steps to bridge the gap in the transition process. The implementation of a follow-up checklist and/or survivorship care plan can be seen as a solution to information needs so care can be confidently transferred to the primary care physicians.

1) Transition Appointment is an effective mechanism to bridge the gap from the oncology setting to the community and the interface between oncology and primary care physician. The appointment can serve to mobilize continuity of care, ensure patients' needs are met and enable patients to become involved in their own healthcare. The transition appointment is often provided by a nurse practitioner, which can enable care to be shifted away from the specialist oncologist, and is a step in the direction of the primary care physician assuming full responsibility of the follow-up care. Saskatchewan, Ontario, Prince Edward Island, Nova Scotia, and Newfoundland incorporate a transition workshop/program and/or classes for survivors during the transition period. These workshops provide patients with basic survivorship information and can help to bridge that gap in transition by empowering patients to become aware and involved in their own care.

2) The Transition Coordinator position is similar to a transition appointment, but it is an individual who works directly with the patient. The role provides support for the patient to manage their own care by providing information on community programs and available resources within the region/area where they live. The transition coordinator is a single point of contact for the patient providing continuous support to the patient through to the survivorship phase. Implementation of a transition coordinator is an important role to help bridge the interface between the oncologist and the primary care provider. This role can act as the glue for continuity of care, ensuring patients' needs are met and/or enabling patients to become involved in their own healthcare.

3) Survivorship Care Plans are tools utilized to improve communication between the specialist oncologist and the primary care physician and the patient (Ganz, Casillas \& Hahn, 
2008; Naidoo et al., 2013). Manitoba, Ontario, Nova Scotia, and Newfoundland currently have some form of a survivorship care plan approach in place, although it is not standardized. The discharge letter and/or transfer of care letter that most provinces utilize as their form of communication often contains some elements of the survivorship care plan and can facilitate improved communication and bridge the gap between the treating oncologist and the primary care physician. The information included in the treatment summary is generally the same in Manitoba, Ontario and Newfoundland and incorporates core elements such as patient demographics, information on the cancer team, surgery (to include date and details), tumour pathology (stage, grade), treatment received and details (chemotherapy, radiation therapy, hormonal therapy), expected side effects (acute and chronic), and diagnostic testing information (for example: date of last mammogram and expected date of next mammogram for breast cancer patients). Information in the follow-up care plan includes signs and symptoms of recurrence and recommended follow-up tests and appointment schedules.

\section{CONCLUSION}

Cancer survivorship is a complex topic that requires communication and coordination between the oncology setting and the community setting to ensure a seamless transition for cancer survivors. This project identified differences in

\section{REFERENCES}

Ackerman, S., Gleaon, N., Monacelli, J., Collado, D., Wang, M., Ho, C., ... Gonzales, R. (2014). When to repatriate? Clinicians' perspectives on the transfer of patient management from specialty to primary care. Journal of General Internal Medicine, 29(10), 1355-1361.

British Columbia Cancer Agency (BCCA). (2015). Survivorship Q primary care program. Retrieved from http://www. bccancer.bc.ca/health-professionals/professional-resources/ survivorship-primary-care

Canadian Cancer Society. (2015). Cancer statistics at a glance. Retrieved from http://www.cancer.ca/en/cancer-information/cancer-101/ cancer-statistics-at-a-glance/?region=on

Cheung, W., Aziz, N., Noone, A., Rowland, J., Potosky, A., Ayanian, J., ... Earle, C. (2013). Physician preferences and attitudes regarding different models of cancer survivorship care: A comparison of primary care providers and oncologists. Journal of Cancer Survivorship, 7, 343-354.

Dawes, A., Hemmelgarn, M., Nguyen, D., Sacks, G., Clayton, S., Cope, J., ... Maggard-Gibbons, M. (2015). Are primary care providers prepared to care for survivors of breast cancer in the safety net? Cancer, 121(8), 1249-1259.

Del Guidice, M., Grunfeld, E., Harvey, B., Piliotis, E., \& Verma, S. (2009). Primary care physicians' views of routine follow-up care of cancer survivors. Journal of Clinical Oncology, 27(20), 3338-3345.

Earle, C., Burstein, H., Winer, E., \& Weeks, J. (2003). Quality of nonbreast cancer health maintenance among elderly breast cancer survivors. Journal of Clinical Oncology, 21(8), 1447-1451.

Earle, C. \& Neville, B. (2004). Underuse of necessary care among elderly colorectal cancer survivors. Cancer, 101(8), 1712-1719.

Fereday, J., \& Muir-Cochrane, E. (2006). Demonstrating rigour using thematic analysis: A hybrid approach of inductive and deductive coding and theme development. International Journal of Qualitative Methods, 5(1), 80-92. transition of care practices across the provinces and yielded some plausible recommendations for high-quality cancer care. There were no real surprises when conducting the environmental scan, as most of the information from the interviews confirmed what was available in the literature. Most interviewees were clearly aware of the growing needs and demands for high-quality follow-up cancer care. However, if survivorship supports/programs are lacking, it is generally due to restricted funding and time. Several interviews ended with, 'I wish we did more', and 'our current system is not good enough; however, we are/may be working towards something better.' Future projects could include 1) information sessions for primary care providers on the complexities of providing adequate follow-up care, and/or 2) implementation of a survivorship program or clinic, which could include a component to track outcomes.

\section{ACKNOWLEDGEMENTS}

I would like to thank the British Columbia Cancer Agency Survivorship and Primary Care Program for their generosity in allowing me to be involved in their wider project; also to Sue Willis of Sheffield Hallam University, Sheffield, UK, for all of her help and support as my supervisor.

\section{CONFLICT OF INTEREST}

The author declares that there are no conflicts of interest.

Ganz, P., Casillas, J., \& Hahn, E. (2008). Ensuring quality care for cancer survivors: Implementing the survivorship care plan. Seminars in Oncology Nursing, 24(3), 208-217.

Government of Canada. (2014). Statistics Canada: Health facts sheet. Ottawa: Statistics Canada.

Grunfeld, E. (2006). Looking beyond survival: How are we looking at survivorship. Journal of Clinical Oncology, 24(32), 5166-5169.

Grunfeld, E., \& Earle, C. (2010). The interface between primary and oncology specialty care: Treatment through survivorship. Journal of National Cancer Institute Monographs, 40, 25-30.

Grunfeld, E., Gray, A., Mant, D., Yudkin, P., Adewuyi-Dalton, R., Coyle, D., et al. (1999). Follow-up of breast cancer in primary care vs specialists care: Results of an economic evaluation. British Journal of Cancer, 79(7/8), 1227-1233.

Grunfeld, E., Levine, M., Julian, J., Coyle, D., Szechtman, B., Mirsky, D., et al. (2006). Randomized control trial of long-term follow-up for early-stage breast cancer: A comparison of family physician versus specialist care. Journal of Clinical Oncology, 24(6), 848-855.

Grunfeld, E., Mant, D., Yudkin, P., Adewuyi-Dalton, R., Cole, D., Stewart, J., et al. (1996). Routine follow-up of breast cancer in primary care: Randomized control trial. British Medical Journal, 313(7058), 665-669.

Grunfeld, E., Yudkin, P., Adewuyi-Dalton, R., Vessey, M., \& Mant, D. (1995). Follow-up in breast cancer. Quality of life unaffected by general practice follow-up. British Medical Journal, 11(6996), 54.

Hagan, T., \& Donovan, H. (2012). Self-advocacy and cancer: A concept analysis. Journal of Advanced Nursing, 69, 2348-2359.

Haq, R., Baker, N., Dastur, D., Leung, F., Leung, E., Li, B., ... Parsons, J. (2013). Designing a multifaceted survivorship care 
plan to meet the information and communication needs of breast cancer patients and their family physicians: Results of a qualitative pilot study. Medical Informatics and Decision Making, 13(76), 1-13.

Mao, J., Bowman, M., Stricker, C.T., DeMichele, A., Jacobs, L., Chan, D., \& Armstrong, K. (2009). Delivery of survivorship care by primary care physicians: The perspective of breast cancer patients. Journal of Clinical Oncology, 27, 933-938.

McCabe, M., \& Jacobs, L. (2012). Clinical update: Survivorship caremodels and programs. Seminars in Oncolog $\gamma$ Nursing, 28(3), e1-e8.

Naidoo, J., Hayes, E., Teo, M., Morgan, A., Calvert, P., \& O’Connor, M. (2013). An Irish breast cancer survivorship study: Are we meeting our patient's needs? Irish Medical Journal, 106(9), 264-266.

Nissen, M., Beran, M., Lee, M., Mehta, S., Pine, D., \& Swenson, K. (2007). Views of primary care providers on follow-up care of cancer patients. Family Medicine, 39, 477-482.

Salz, T., Oeffinger, K.C., Lewis, P.R., Williams, R.L., Rhyne, R.L., \& Yeazel, M.W. (2012). Primary care providers' needs and preferences for information about colorectal cancer survivorship care. Journal of the American Board of Family Medicine, 25, 635-651.

Sisler, J., Brown, J., \& Stewart, M. (2004). Family physicians' roles in cancer care: Survey of patients on a provincial cancer registry. Canadian Family Physician, 50, 889-896.
Smith, S., Singh-Carlson, S., Downie, L., Payeur, N., \& Wai, E. (2011). Survivors of breast cancer: Patient perspectives on survivorship care planning. Journal of Cancer Survivorship, 5, 337-344.

Snyder, C., Earle, C., Herbert, R., Neville, B., Blackford, A., \& Frick, K. (2008). Preventative care for colorectal cancer survivors: A 5-year longitudinal study. Journal of Clinical Oncology, 26(7), 1073-1079.

Urquhart, R., Folkes, A., Babineau, J., \& Grunfeld, E. (2012). Views of breast and colorectal cancer survivors on their routine follow-up care. Current Oncology, 19(6), 294-301.

Wattchow, D., Weller, D., Esterman, A., Pilotto, L., McGorm, K., Hammett, Z., ... Silagy, C. (2006). General practice vs surgicalbased follow-up for patients with colon cancer: Randomised controlled trial. British Journal of Cancer, 94(8), 1116-1121.

Wiljer, D., Urowitz, S., Jones, J., Kornblum, A., Secord, S., \& Catton, P. (2013). Exploring the use of the survivorship consult in providing survivorship care. Support Care Cancer, 21, 2117-2124.

Wood, M.L. (1993). Communication between cancer specialists and family doctors. Canadian Family Physician, 39, 49-57.

Ye, A., Cheung, W., Goddard, K., Horvat, D., \& Olson, R. (2015). Follow-up patterns of cancer survivors: A survey of Canadian radiation oncologists. Journal of Cancer Survivorship, 9, 388-403. 